\title{
ALDH2 Inhibition Potentiates High Glucose Stress-Induced Injury in Cultured Cardiomyocytes
}

\author{
Guodong Pan, ${ }^{1}$ Mandar Deshpande, \\ Rajarajan A. Thandavarayan, ${ }^{2}$ and Suresh Selvaraj Palaniyandi ${ }^{1,3}$ \\ ${ }^{1}$ Division of Hypertension and Vascular Research, Department of Internal Medicine, Henry Ford Health System, \\ Detroit, MI 48202, USA \\ ${ }^{2}$ Department of Cardiovascular Sciences, Center for Cardiovascular Regeneration, Houston Methodist Research Institute, \\ Houston, TX, USA \\ ${ }^{3}$ Department of Physiology, Wayne State University, Detroit, MI 48202, USA
}

Correspondence should be addressed to Suresh Selvaraj Palaniyandi; spalani2@hfhs.org

Received 20 April 2016; Revised 26 July 2016; Accepted 22 August 2016

Academic Editor: Kim Connelly

Copyright (C) 2016 Guodong Pan et al. This is an open access article distributed under the Creative Commons Attribution License, which permits unrestricted use, distribution, and reproduction in any medium, provided the original work is properly cited.

\begin{abstract}
Aldehyde dehydrogenase (ALDH) gene superfamily consists of 19 isozymes. They are present in various organs and involved in metabolizing aldehydes that are biologically generated. For instance, ALDH2, a cardiac mitochondrial ALDH isozyme, is known to detoxify 4-hydroxy-2-nonenal, a reactive aldehyde produced upon lipid peroxidation in diabetic conditions. We hypothesized that inhibition of ALDH leads to the accumulation of unmetabolized 4HNE and consequently exacerbates injury in cells subjected to high glucose stress. H9C2 cardiomyocyte cell lines were pretreated with $10 \mu \mathrm{M}$ disulfiram (DSF), an inhibitor of ALDH2 or vehicle (DMSO) for 2 hours, and then subjected to high glucose stress $\{33 \mathrm{mM} \mathrm{D}$-glucose (HG) or $33 \mathrm{mM} \mathrm{D}$-mannitol as an osmotic control (Ctrl)\} for $24 \mathrm{hrs}$. The decrease in ALDH2 activity with DSF pretreatment was higher in HG group when compared to Ctrl group. Increased $4 \mathrm{HNE}$ adduct formation with DSF pretreatment was higher in HG group compared to Ctrl group. Pretreatment with DSF leads to potentiated HG-induced cell death in cultured H9C2 cardiomyocytes by lowering mitochondrial membrane potential. Our results indicate that ALDH2 activity is important in preventing high glucose induced cellular dysfunction.
\end{abstract}

\section{Introduction}

ALDH is a family of multiple homotetrameric enzymes [1]. As the name implies, ALDH enzymes metabolize aldehydes into carboxylic acids. These aldehydes include aliphatic and aromatic aldehydes from the environment, food, and those generated during cell metabolism. ALDH plays a major role in detoxifying reactive aldehydes. When ALDH2 was overexpressed, the deleterious effects of acetaldehyde in the heart were diminished [2]. A single point mutation in ALDH2 greatly reduces ALDH2 activity in East Asians, which leads to accumulation of acetaldehyde and ultimately facial flushing [3] and other harmful effects [4]. Moreover, this mutation is reportedly associated with diabetic complications [5] and coronary diseases in East Asians. It is suggested that the toxicity of reactive aldehydes plays main role in the pathogenesis.

Hyperglycemia-induced oxidative stress is implicated in the pathogenesis of diabetic complications [6]. We focus on the secondary products of oxidative stress, that is, reactive aldehydes. Toxic reactive aldehydes such as 4-hydroxy-2nonenal (4HNE) are produced by lipid peroxidation during oxidative stress in the mitochondria [7]. 4HNE is especially toxic as it forms adducts with proteins and leads to cellular dysfunction [7-14].

In another recent report, we have shown that pharmacological inhibition of ALDH2 per se induces mitochondrial dysfunction and cell death [15]. To provide an extension of our previous results, we planned to investigate the effect of ALDH2 inhibition using disulfiram (DSF), an ALDH2 
inhibitor, on high glucose stress in H9C2 cardiomyocyte cell lines.

\section{Materials and Methods}

2.1. H9C2 Cell Culture and Treatment Protocol. H9C2 cell lines \{ATCC CLR-1446, routinely used as cardiomyocytes [16-18]\}, were cultured and grown in DMEM medium. The cells were grown to reach $60-70 \%$ confluence and subsequently used in the experiments.

After $1 \mathrm{hr}$ of starving, H9C2 cells were treated with DSF (Santa Cruz Chemicals, Santa Cruz, CA), an inhibitor of ALDH2 or DMSO for 2 hours, and then subjected to high glucose stress for 24 hours. The high glucose stress was induced by replacing the culture medium with $33 \mathrm{mM}$ of D-glucose (HG). We maintained the glucose concentration in the cell culture medium throughout the study period by measuring the glucose level using a glucometer. To nullify the osmotic stress caused by high concentration of solute in the culture medium is not the cause for the observed cellular stress, the culture medium of another set of cells was replaced with the same $33 \mathrm{mM}$ D-mannitol (Ctrl).

We chose 2 doses for DSF, that is, $10 \mu \mathrm{M}$ and $2 \mu \mathrm{M}$ after performing a dose-response curve using DSF in inhibiting ALDH2 activity as shown in Supplementary Figure 1 (see Supplementary Material available online at http://dx.doi.org/ $10.1155 / 2016 / 1390861)$. We used $2 \mu \mathrm{M}$ for the mitochondrial studies as mitochondrial oxygen consumption rate (OCR) was decreased below detection level with $10 \mu \mathrm{M}$ DSF treatment.

2.2. Intracellular ROS Measurement. Intracellular ROS can oxidize the nonfluorescent 29,79-dichlorofluorescein diacetate (DCFH-DA) to generate fluorescent 29,79-dichlorofluorescein (DCF). Fluorescence intensity of DCF can be measured using a fluorimeter. Therefore to measure intracellular ROS in H9C2 cells, we used the DCF kit and followed the manufacturer's instructions (Abcam Inc., Cambridge, MA). Briefly, cells were washed twice with PBS after the study protocol, and then the ROS detection solution was added. Cells were stained at $37^{\circ} \mathrm{C}$ in the dark for $20 \mathrm{~min}$. The fluorescence intensity of DCF was measured using a fluorimeter at $504 \mathrm{~nm}$ excitation and $524 \mathrm{~nm}$ emission wavelengths.

2.3. ALDH Activity Assay. ALDH2 activity was measured by following the procedure described elsewhere [19, 20]. In brief, enzymatic activity of ALDH2 from cell lysate was determined spectrophotometrically by using the reductive reaction of $\mathrm{NAD}+$ to $\mathrm{NADH}$ at $\lambda 340 \mathrm{~nm}$. All assays were carried out at $25^{\circ} \mathrm{C}$ in $0.1 \mathrm{M}$ sodium pyrophosphate buffer, $\mathrm{pH}=9.5$ with $2.4 \mathrm{mM} \mathrm{NAD}+$ as a cofactor and $10 \mathrm{mM}$ acetaldehyde as the substrate.

2.4. Western Blot Analysis of $4 \mathrm{HNE}$ Protein Adducts. $4 \mathrm{HNE}$ protein adducts were evaluated by Western immunoblot as described earlier [21, 22]. In brief, protein samples from cell lysates were separated using SDS-PAGE and the proteins were then transferred to immobilon- $\mathrm{P}$ membranes (Millipore, Billerica, MA). Anti-4HNE-Cys/His/Lys rabbit antibody (Millipore) and anti- $\beta$-actin mouse monoclonal antibody (SCBT, Santa Cruz, CA) at a concentration of 1:1000 ( $4^{\circ} \mathrm{C}$ overnight) were used. The bound antibody was visualized with horseradish peroxidase- (HRP-) coupled secondary antibody and chemiluminescence detection reagents.

Intensity of scanned Western blot images was analyzed using Image J software. The ratios of levels of $4 \mathrm{HNE}$ protein adducts and $\beta$-actin, the loading control, were calculated and plotted as graphs.

\subsection{Trypan Blue Exclusion Cell Death Assay in H9C2 Car-} diomyocytes. Cells grown at 60-70\% confluence were starved for $1 \mathrm{hr}$ using DMEM media without serum. The plates were then divided into the following treatment groups $(n=6$ plates each). At the end of the protocol, cells were washed with TBS buffer and trypsinized. Trypan blue was added at a concentration of $1: 1$ and subsequently an automated cell counter (Bio-Rad) was used to determine \% of live cells remaining.

2.6. Mitochondrial ROS Measurement. MitoSOX reagent was used to measure mitochondrial ROS as explained in the manufacturer's instructions. $1.0-2.0 \mathrm{~mL}$ of $5 \mu \mathrm{M}$ MitoSOX reagent working solution was used to cover $\mathrm{H} 9 \mathrm{c} 2$ cells and incubated for 10 minutes at $37^{\circ} \mathrm{C}$, protected from light. After washing cells with warm buffer, we processed the cells for imaging using florescent microscope with absorption at $510 \mathrm{~nm}$ and emission $580 \mathrm{~nm}$. We also quantified the fluorescence using Synergy H1 Multi-Mode Reader (BioTek Inc.).

2.7. Measurement of Mitochondrial Transmembrane Potential. $5,5^{\prime}, 6,6^{\prime}$-Tetrachloro-1,1',3,3' -tetraethylbenzimidazolyl-

carbocyanine iodide (JC-1; Sigma-Aldrich) was used to determine changes in mitochondrial transmembrane potential $\left(\Delta \Psi_{\mathrm{m}}\right)$. JC-1 $(10 \mu \mathrm{L} ; 200 \mu \mathrm{M}$; final concentration $2 \mu \mathrm{M})$ was added to $\mathrm{H} 9 \mathrm{c} 2$ cells on coverslips, incubated for $45 \mathrm{~min}$ in the dark, and washed twice with PBS. JC1 fluorescence was measured from a single excitation wavelength $(490 \mathrm{~nm})$ with dual emission (shift from green at $530 \mathrm{~nm}$ to red at $590 \mathrm{~nm}$ ) using a fluorimeter. Loss of mitochondrial $\Delta \Psi_{\mathrm{m}}$ is presented as the relative ratio of green to red fluorescence.

2.8. Measurement of Oxygen Consumption Rate (OCR) in H9C2 Cardiomyocytes. H9C2 cells were seeded at 30,000 cells/well onto Bioscience V7 culture plates in growth medium containing 15\% FBS (fetal bovine serum) on the first day. The next day, medium was replaced, and cells were grown in the culture growth medium. Within $24 \mathrm{hrs}$ of seeding, a confluent monolayer of H9C2 cells was formed and then cells were used as described below. XF24 Analyzer (Seahorse Biosciences) was used to measure OCR to be monitored in real time function in H9C2 cardiomyocytes as mentioned in our earlier report. For all measurements, the culture medium was changed to serum-free media for $1 \mathrm{hr}$ prior to assay. At the end of the treatment, cells were washed with buffer and Cell Mito Stress Test (CMST) media for cell bioenergetics measurements were added into each well.

The plate was then transferred to the XF24 instrument and the experiment was initiated. Oligomycin, an ATP 


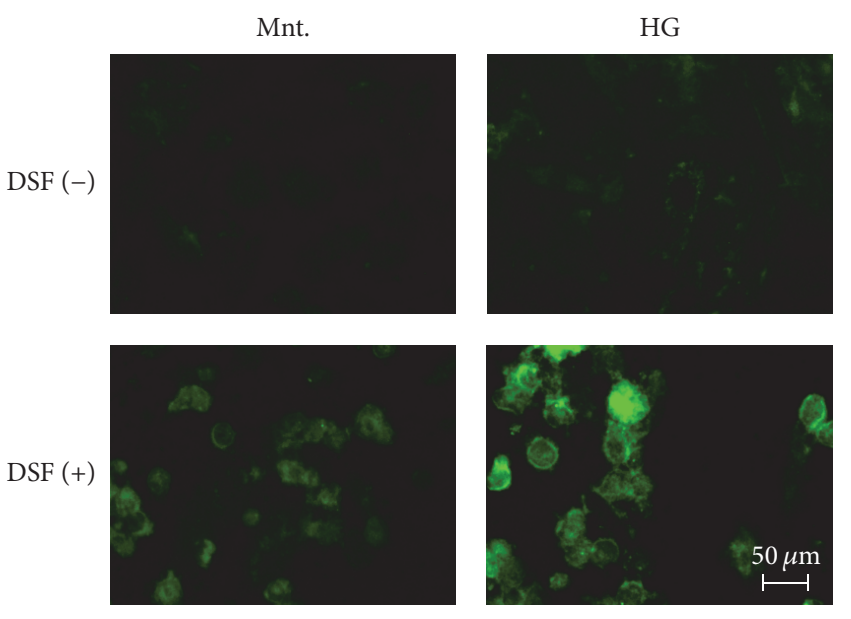

(a)

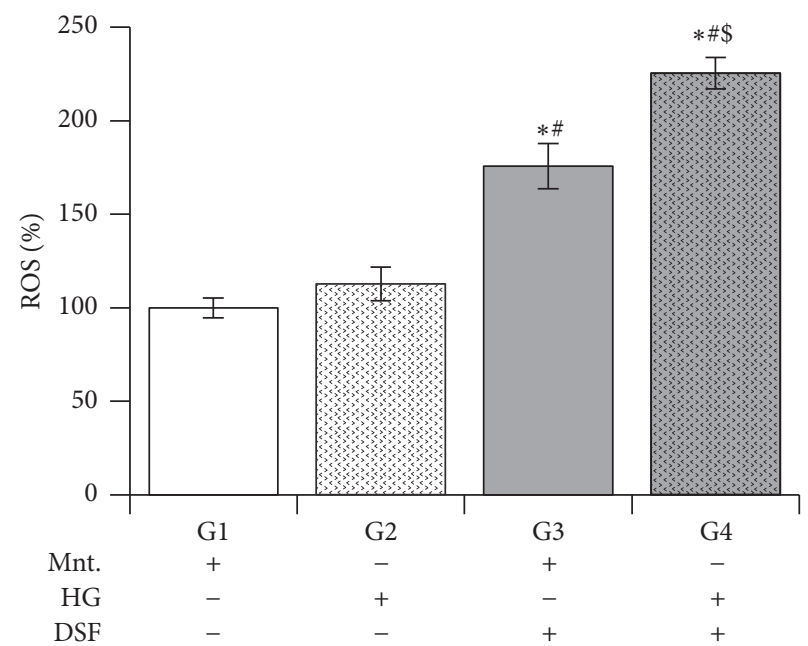

(b)

FIGURE 1: ALDH2 inhibition by pretreating with disulfiram (DSF) and reactive oxygen species (ROS) levels. (a) Representative photomicrographs of DCF stained cells from respective treatments. (b) Increase in ROS in cultured H9C2 cardiomyocytes subjected to high glucose stress (G2) compared to equimolar mannitol (G1). Disulfiram (DSF) pretreatment increased ROS levels in both mannitol (G3) and high glucose (G4) groups. The data expressed are mean \pm SEM. $N=4-6 .{ }^{*} p<0.05$ versus G1, ${ }^{\#} p<0.05$ versus G2, and ${ }^{\$} p<0.05$ versus G3.

synthase inhibitor (to check the ATP mediated respiration); FCCP, a known uncoupler and proton translocating ionophore (to determine maximal respiration and the mitochondrial reserve capacity); and antimycin A, an inhibitor of the mitochondrial electron transport chain (to check OCR from nonmitochondrial respiration related) prepared in CMST were injected sequentially through ports in the Seahorse Flux Pak cartridges and then mitochondrial OCR was recorded.

2.9. Statistical Analysis. Data is presented as mean \pm standard error of the mean (SEM). One-way ANOVA was used to compare the groups. Statistical significance was achieved when $p$ was $<0.05$.

\section{Results}

3.1. Effect of DSF Pretreatment on ROS Levels in Cultured H9C2 Cardiomyocytes Subjected to High Glucose Stress. DSF pretreatment significantly increased ROS levels in cultured H9C2 cardiomyocytes subjected to high glucose stress compared to DSF-untreated cells presented with equimolar concentrations of glucose or mannitol. Surprisingly, DSF pretreatment significantly increased ROS levels in the mannitol group compared to the DSF-untreated group. However, DSF pretreatment enhanced the ROS increase significantly higher in $\mathrm{H} 9 \mathrm{C} 2$ cells with high glucose stress compared to cells in the control (mannitol) group (Figure 1). H9C2 Cardiomyocytes Subjected to High Glucose Stress. DSF

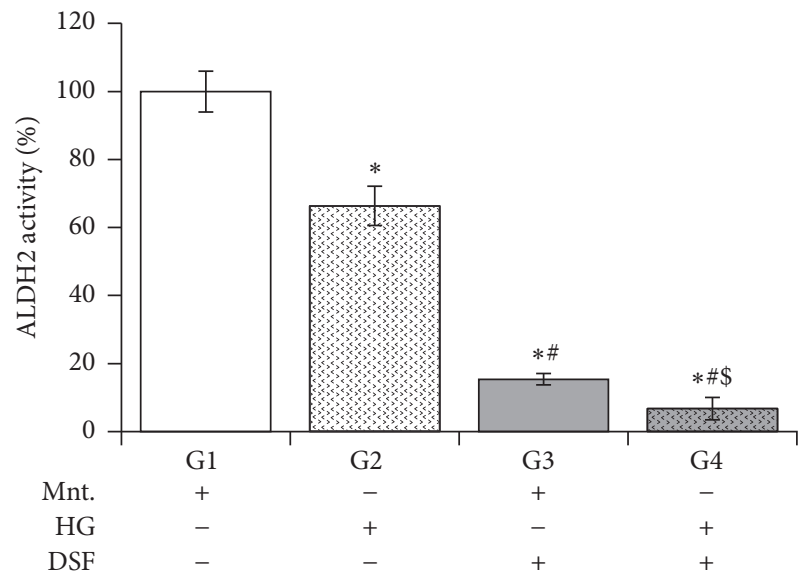

FIGURE 2: Disulfiram (DSF) pretreatment and ALDH2 activity. DSF $10 \mu \mathrm{M}$ reduces ALDH2 activity in both HG and Ctrl groups. The data expressed are mean \pm SEM. $N=4-6$. G1, G2, G3, and G4 depict mannitol, high glucose stress, DSF + mannitol, and DSF + high glucose stress. G1, G2, G3, and G4 depict mannitol, high glucose stress, DSF + mannitol, and DSF + high glucose stress, respectively. ${ }^{*} p<0.05$ versus G1, ${ }^{*} p<0.05$ versus $\mathrm{G} 2$, and ${ }^{\$} p<0.05$ versus $\mathrm{G} 3$.

pretreatment significantly reduced ALDH2 activity in cultured H9C2 cardiomyocytes subjected to high glucose stress compared to DSF-untreated cells presented with equimolar concentrations of glucose or mannitol.

Furthermore, this DSF pretreatment significantly reduced ALDH2 activity in $\mathrm{H} 9 \mathrm{C} 2$ cells with high glucose stress compared to cells in the control (mannitol) group (Figure 2). 


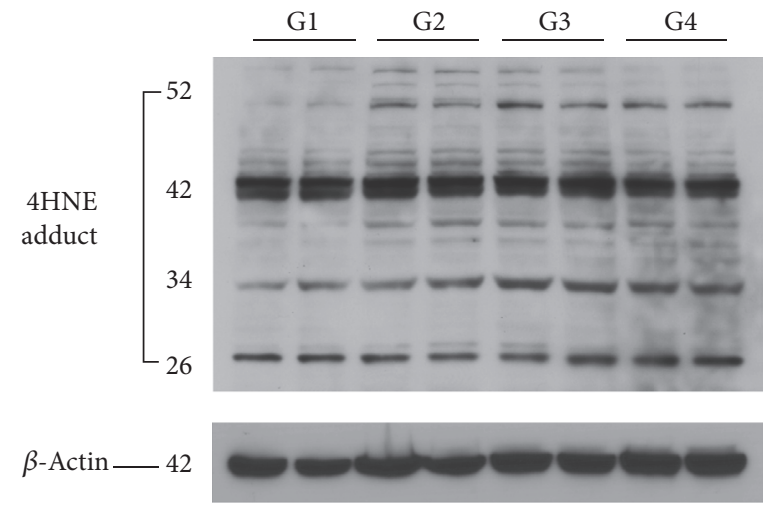

(a)

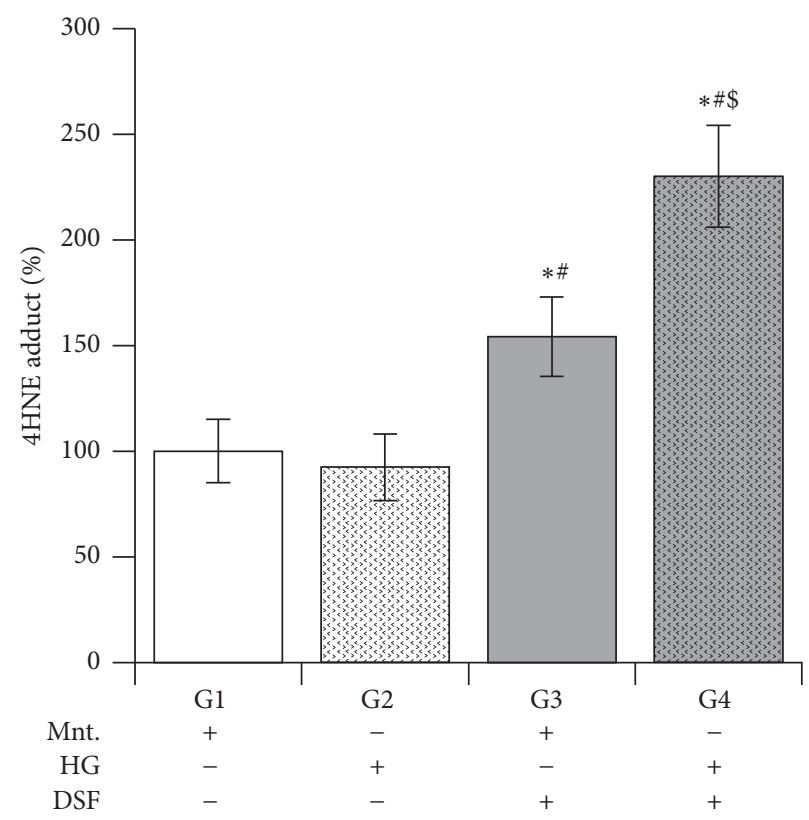

(b)

FIGURE 3: Disulfiram (DSF) pretreatment and 4HNE protein adducts. (a) Western blot images of 4HNE adducts were shown. Beta-actin was used as a loading control. (b) The quantification data of Western immunoblot. Disulfiram (DSF) (10 $\mu \mathrm{M})$ treatment increases $4 \mathrm{HNE}$ protein adduct formation; the data expressed are mean \pm SEM. $N=4-6$. G1, G2, G3, and G4 depict mannitol, high glucose stress, DSF + mannitol, and DSF + high glucose stress, respectively. ${ }^{*} p<0.05$ versus G1, ${ }^{*} p<0.05$ versus $\mathrm{G} 2$, and ${ }^{\$} p<0.05$ versus G3.

\subsection{Effect of DSF Treatment on 4HNE Adducts Formation} in Cultured H9C2 Cardiomyocytes Subjected to High Glucose Stress. DSF pretreatment for 2 hours significantly augmented $4 \mathrm{HNE}$ adducts formation in cells subjected to high glucose stress compared to DSF-untreated cells presented with equimolar concentrations of glucose or mannitol in their culture medium. Moreover, among the DSF-pretreated cells, increase in $4 \mathrm{HNE}$ adduct formation was found at a significantly higher amount in the HG group compared to the Ctrl group (Figure 3).

3.4. Effect of DSF Treatment on Cell Death in Cultured H9C2 Cardiomyocytes Subjected to High Glucose Stress. High glucose stress induced cell death in cultured H9C2 cardiomyocytes. Pretreatment with DSF significantly increased cell death in the high glucose group compared to the untreated high glucose group. Surprisingly, DSF pretreatment significantly increased cell death in the mannitol group compared to cells in the DSF-untreated group. Among the DSF treated groups, cell death was significantly increased in the HG group compared to the mannitol group (Figure 4).

\subsection{Effect of DSF Treatment on Mitochondrial Membrane} Potential in Cultured H9C2 Cardiomyocytes Subjected to High Glucose Stress. Pretreatment with DSF reduced mitochondrial membrane potential in the HG group compared to the DSF-untreated HG and Ctrl groups. Furthermore, DSF enhanced the reduction in mitochondrial membrane potential in cultured H9C2 cardiomyocytes subjected to high glucose stress compared to cells presented with equimolar mannitol in their culture medium (Figure 5).
HG increased mitochondrial ROS in cultured H9C2 cardiomyocytes which was further enhanced in both Ctrl and HG groups with DSF pretreatment (Supplementary Figure 2).

3.6. Effect of DSF Treatment on Mitochondrial Respiration in Cultured H9C2 Cardiomyocytes Subjected to High Glucose Stress. The mitochondrial OCR was measured to determine mitochondrial respiration. Basal and FCCP-induced maximal OCR were decreased in HG group compared to Ctrl group. Pretreatment with DSF enhanced maximal OCR reduction significantly in $\mathrm{HG}$ group but not in Ctrl group (Figure 6).

\section{Discussion}

In this study, we report that the pharmacological inhibition of ALDH2 potentiates high glucose induced deleterious effects in cultured H9C2 cardiomyocyte cell lines.

In a recent study, we reported that ALDH2 inhibition by $75 \mu \mathrm{M}$ DSF per se attenuated mitochondrial respiration and induced cell death in cultured H9C2 cardiomyocyte cell lines without any stress [15]. While DSF inhibited recombinant mitochondrial ALDH2 enzyme with an $\mathrm{IC}_{50}$ value of $36.4 \mu \mathrm{M}$ [23], we found that DSF $75 \mu \mathrm{M}$ actually attenuated $50 \%$ of ALDH2 activity in H9C2 cells without any stress [15]. However the treatment duration was only 2-4 hours without any stress. In this study, we plan to pretreat cells with DSF in high glucose stress. In order to do this, we performed a pilot study to determine the DSF dose as explained in the method section. We first inhibited ALDH2 activity in H9C2 


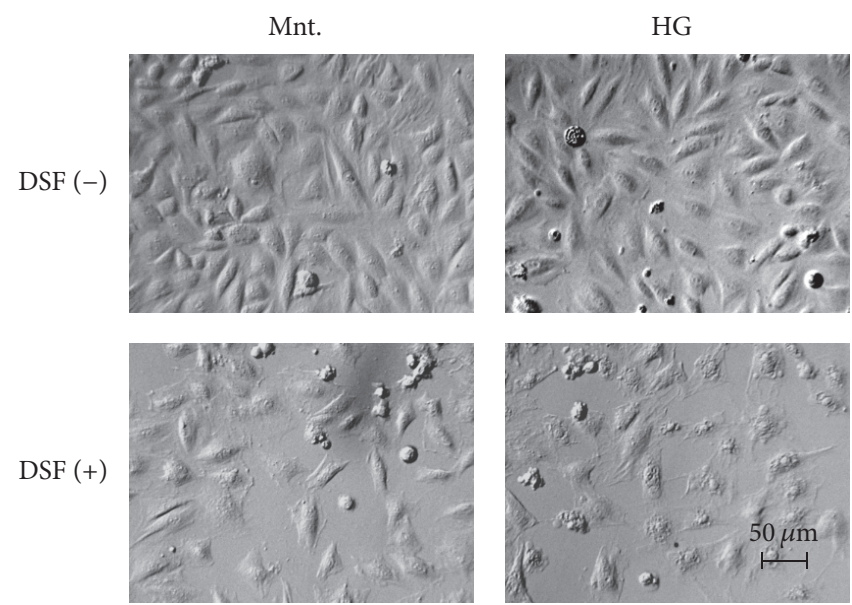

(a)

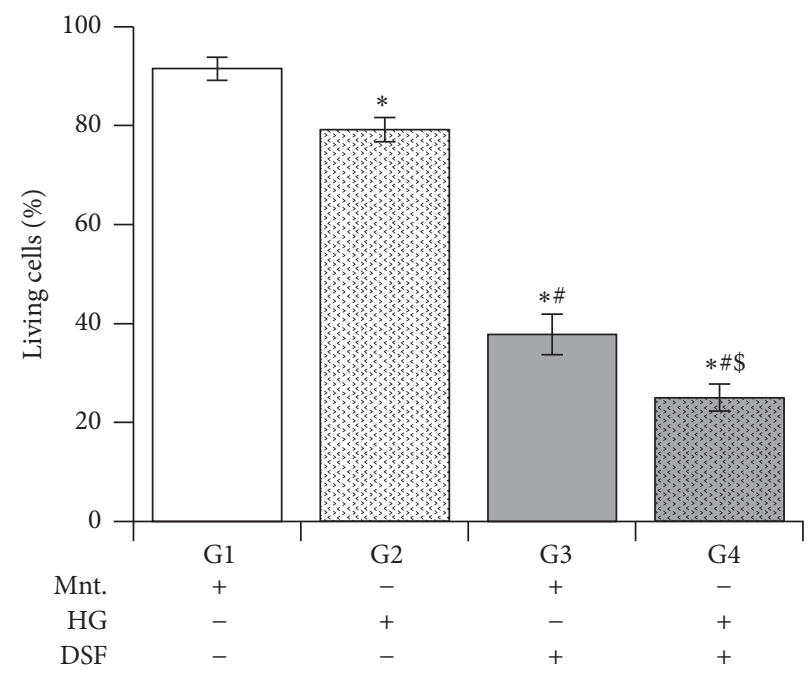

(b)

FiguRE 4: Disulfiram (DSF) pretreatment and cell death. (a) Representative Hoffman modulation-contrast microscopic images of cells were shown. The normal cell morphology was maintained in mannitol with no DSF. However, the cells from all other 3 groups were depicting cell death (shape altered with cell damage) from all 4 groups in various degrees. In the HG and DSF group, lot of cells show cell disintegration depicting cell death. (b) DSF $10 \mu \mathrm{M}$ treatment leads to cell death cultured H9C2 cardiomyocytes subjected to high glucose stress (HG) compared to equimolar mannitol (Ctrl). Trypan exclusion assay was used to measure the cell death. The data expressed are mean \pm SEM. $N=$ 4-6. G1, G2, G3, and G4 depict mannitol, high glucose stress, DSF + mannitol, and DSF + high glucose stress, respectively. ${ }^{*} p<0.05$ versus G1, ${ }^{\#} p<0.05$ versus $\mathrm{G} 2$, and ${ }^{\$} p<0.05$ versus $\mathrm{G} 3$.
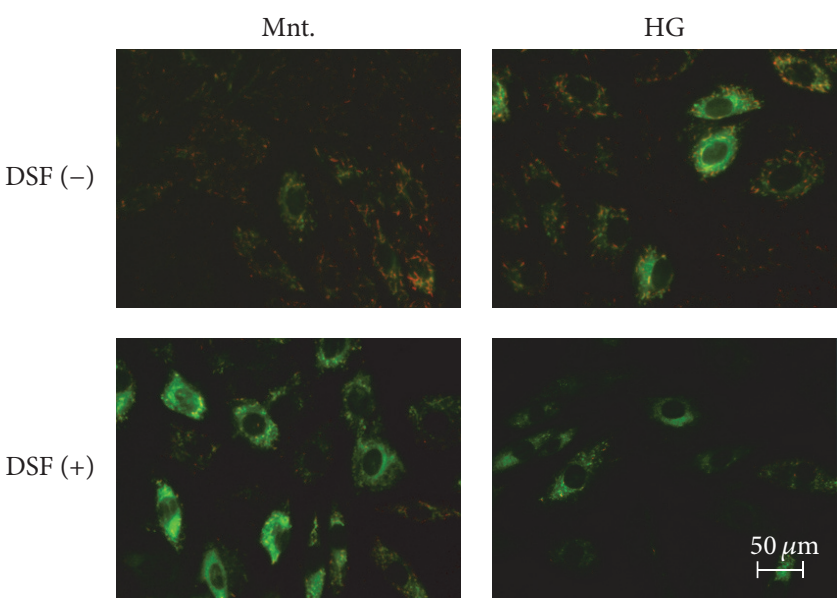

(a)

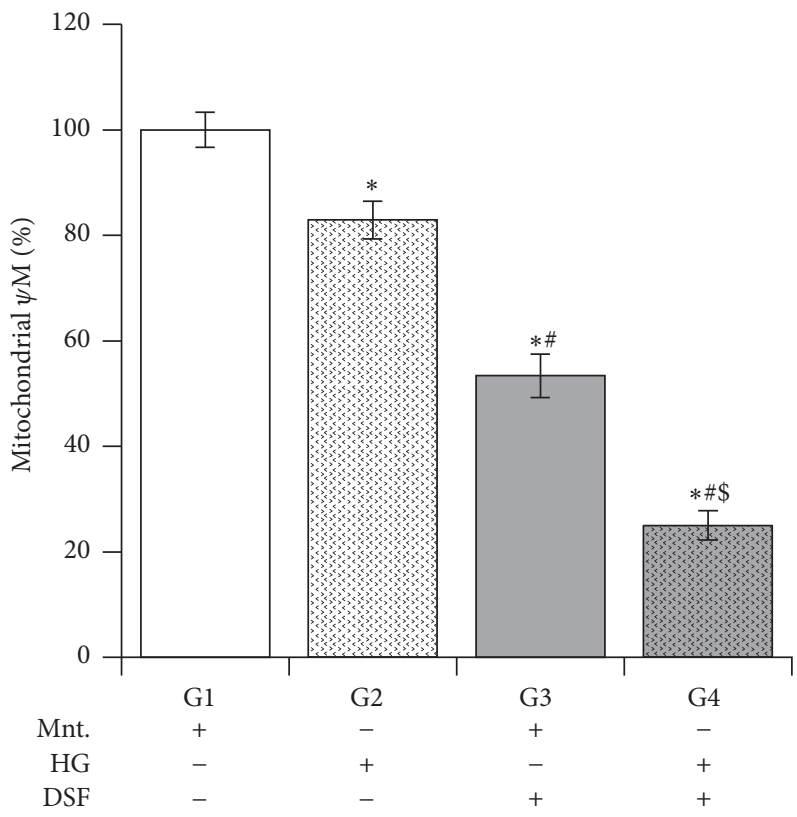

(b)

FIGURE 5: Disulfiram (DSF) pretreatment and mitochondrial membrane potential. (a) Representative microscopic images of cells with JC-1 dye to measure mitochondrial membrane potential $(\Psi)$. Increase in red to green florescence ratio indicates high membrane potential as shown in mannitol group, while increased green florescence indicates drop in mitochondrial $\Psi$. (b) DSF $10 \mu \mathrm{M}$ treatment reduces mitochondrial $\Psi$ when measured using JC-1 dye in cultured H9C2 cardiomyocytes subjected to high glucose stress (HG) compared to equimolar mannitol (Ctrl). The data expressed are mean \pm SEM. $N=4-6$. G1, G2, G3, and G4 depict mannitol, high glucose stress, DSF + mannitol, and DSF + high glucose stress, respectively. ${ }^{*} p<0.05$ versus G1, ${ }^{*} p<0.05$ versus $\mathrm{G} 2$, and ${ }^{\$} p<0.05$ versus G3. 


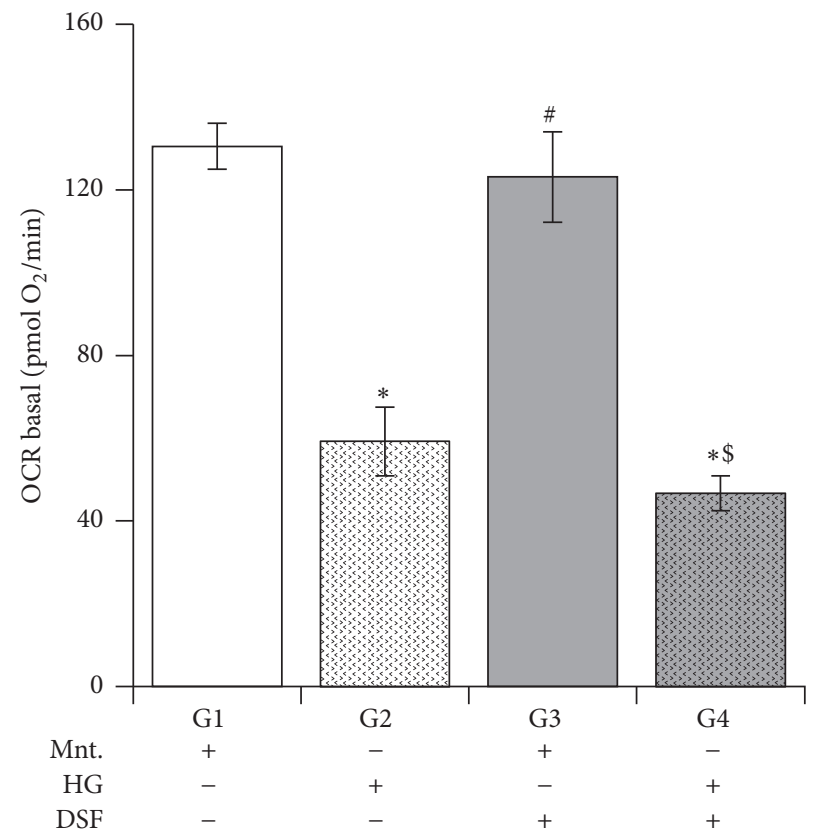

(a)

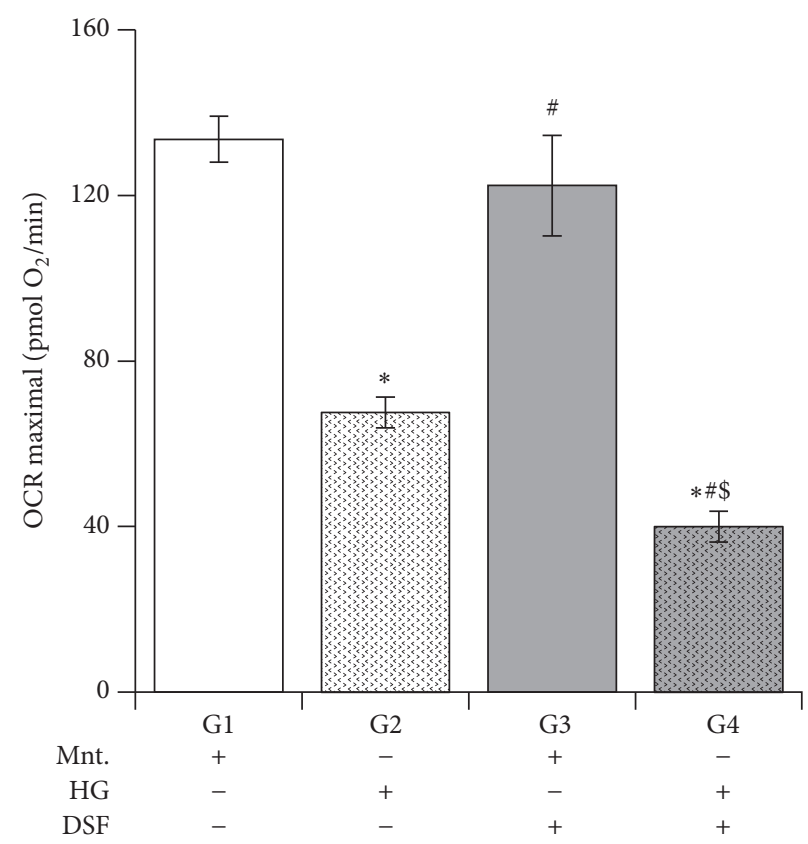

(b)

FIGURE 6: Disulfiram (DSF) pretreatment and mitochondrial oxygen consumption rate (OCR). (a) Basal mitochondrial OCR from G1, G2, G3, and G4 which depict mannitol, high glucose stress, DSF + mannitol, and DSF + high glucose stress. $N=4-6$. (b) Maximal mitochondrial OCR with FCCP treatment. G1, G2, G3, and G4 depict mannitol, high glucose stress, DSF + mannitol, and DSF + high glucose stress, respectively. $N=4-6 .{ }^{*} p<0.05$ versus G1, ${ }^{*} p<0.05$ versus $\mathrm{G} 2$, and ${ }^{\$} p<0.05$ versus G3.

cells using DSF and then subjected them to high glucose stress. To induce high glucose stress in vitro the cell culture medium was replaced with $33 \mathrm{mM}$ D-glucose or $33 \mathrm{mM} \mathrm{D}$ mannitol as per previous reports [24]. As we explained in our previous report [15], H9C2 cardiomyocyte cell lines are suitable alternatives for primary cardiomyocytes to be used in hyperglycemic studies. In fact, it was shown that isolated cardiomyocytes and $\mathrm{H} 9 \mathrm{C} 2$ cells respond similarly under high glucose stress [25]. Using H9C2 cells was a choice that is logically supported.

We and others reported that hyperglycemia decreases myocardial ALDH2 activity in rodents $[19,26,27]$. The possible mechanism that we propose for this reduction is that hyperglycemia increases ROS generation which attenuates ALDH2 activity and thereby increases $4 \mathrm{HNE}$ adduct formation, which ultimately leads to cellular dysfunction and then death. Increased ROS in type 1 and type 2 diabetes is implicated in the development of diabetic cardiomyopathy $[28,29]$.

DSF pretreatment significantly increased ROS levels in H9C2 cells with mannitol. This result suggests that ALDH2 inhibition by DSF itself can induce ROS generation independent of high glucose stress. DSF treatment further enhanced the increase in ROS levels in high glucose stress compared to control. DSF pretreatment reduced ALDH2 activity more in cells with high glucose compared to control. It appears DSF pretreatment made the cells more susceptible to high glucose mediated ALDH2 impairment. Next, we found a significant increase in 4HNE protein adducts only in DSF-pretreated cells with high glucose stress. This increase was not present in the control group or the DSF-untreated HG group. Though we observed increased ROS levels and reduced ALDH2 activity in the control group with DSF treatment, we did not observe an increase in the level of $4 \mathrm{HNE}$ protein adducts. The exact mechanism for this discrepancy is unknown. However we suppose the $20 \%$ ALDH2 activity remaining may be sufficient to metabolize $4 \mathrm{HNE}$ in the control group. Perhaps, this could have caused the observed reduction in the level of $4 \mathrm{HNE}$ protein adducts [30].

The mitochondrial membrane potential was reduced in the DSF treated HG group compared to the DSF-untreated HG group and DSF treated control group. It unequivocally suggests that DSF increases the susceptibility in cells subjected to high glucose stress to a drop in mitochondrial membrane potential despite the difference in glucose levels. Progressive decrease in mitochondrial transmembrane potential is associated with apoptotic cell death [31]. In our study, there was a decrease in the mitochondrial potential under the hyperglycemic condition, suggesting that hyperglycemia could act as an apoptosis-inducing stimulus. Therefore, an intact transmembrane and its potential are indispensable for normal mitochondrial function.

Next, we found mitochondrial OCR at basal and FCCPinduced maximal response were reduced in HG stress. DSF further augmented the reduction in OCR. As per our earlier report, ALDH2 inhibition per se attenuated OCR and further this reduction in OCR is linked with cell death [15].

High glucose alone caused 20\% cell death. But DSF pretreatment increased the cell death to more than $90 \%$ in the high glucose group. Surprisingly, DSF increased cell death 
to $80 \%$ in control cells as well. This data again implicates the role of ALDH2 inhibition in maintaining cell viability and potentiating high glucose mediated cell death. This was also seen in our earlier study as ALDH2 inhibition by DSF and ALDH2 siRNA contributed to cell death by decreasing mitochondrial function in $\mathrm{H} 9 \mathrm{C} 2$ cells during 2-4 hours [15]. Decrease in ALDH2 activity by DSF without causing $4 \mathrm{HNE}$ adducts formation itself was noted to cause significant reduction in mitochondrial reserve capacity and subsequently cell death in H9C2 cells [15]. Similarly we observed an increase in cell death in control cells perhaps due to the reduction in mitochondrial respiratory defects demonstrated previously [15]. Strikingly, the ALDH2 inhibition by DSF led to a significant increase in $4 \mathrm{HNE}$ adducts as well as a decrease in mitochondrial membrane potential in cells under high glucose stress. Earlier reports have shown that hyperglycemia reduced the activity and levels of myocardial ALDH2 [27, 32]. Overexpression of ALDH2 conferred cardioprotection by attenuating $4 \mathrm{HNE}$ toxicity in STZ-injected hyperglycemic mice [32]. Taken altogether, we suggest ALDH2 activity plays an important role in the process of hyperglycemiainduced cardiac damage. Similarly we speculate that the hyperglycemic stress in ALDH2 2 carriers with diabetes or noncarriers with other comorbidities with low ALDH2 activity may be vulnerable to cardiac tissue damage. Our previous observation points out that long-term diabetes in patients reduces ALDH2 activity and increases $4 \mathrm{HNE}$ adduct formation in diabetic hearts [26]. It also implies that chronic hyperglycemia can lead to an inevitable decrease in ALDH2 activity and cause an exacerbation of hyperglycemia-induced cardiac damage.

We conclude this report by stating that the inhibition of ALDH2 activity potentiates high glucose stress-induced deleterious effects in cardiac cells. Our study implicates individuals with compromised ALDH2 activity may have higher propensity to develop diabetic cardiac complications or increase the severity of the damage.

\section{Competing Interests}

The authors declare that there is no conflict of interests.

\section{Acknowledgments}

This work was partially supported by AHA Scientist Development Grant 14SDG20050030 (Suresh Selvaraj Palaniyandi) and internal grant from Henry Ford Health System (Suresh Selvaraj Palaniyandi).

\section{References}

[1] B. Jackson, C. Brocker, D. C. Thompson et al., "Update on the aldehyde dehydrogenase gene $(A L D H)$ superfamily," Human Genomics, vol. 5, no. 4, pp. 283-303, 2011.

[2] T. A. Doser, S. Turdi, D. P. Thomas, P. N. Epstein, S.-Y. Li, and J. Ren, "Transgenic overexpression of aldehyde dehydrogenase2 rescues chronic alcohol intake-induced myocardial hypertrophy and contractile dysfunction," Circulation, vol. 119, no. 14, pp. 1941-1949, 2009.
[3] S. Harada, D. P. Agarwal, and H. W. Goedde, "Aldehyde dehydrogenase deficiency as cause of facial flushing reaction to alcohol in Japanese," The Lancet, vol. 318, no. 8253, p. 982, 1981.

[4] P. J. Brooks, M.-A. Enoch, D. Goldman, T.-K. Li, and A. Yokoyama, "The alcohol flushing response: an unrecognized risk factor for esophageal cancer from alcohol consumption," PLoS Medicine, vol. 6, no. 3, Article ID e1000050, 2009.

[5] Y. Suzuki, M. Taniyama, T. Muramatsu et al., "ALDH2/ADH2 polymorphism associated with vasculopathy and neuropathy in type 2 diabetes," Alcoholism: Clinical and Experimental Research, vol. 28, no. 8, 2004.

[6] M. Brownlee, "The pathobiology of diabetic complications: a unifying mechanism," Diabetes, vol. 54, no. 6, pp. 1615-1625, 2005.

[7] V. R. Mali and S. S. Palaniyandi, "Regulation and therapeutic strategies of 4-hydroxy-2-nonenal metabolism in heart disease," Free Radical Research, vol. 48, no. 3, pp. 251-263, 2014.

[8] I. E. Blasig, T. Grune, K. Schonheit et al., "4-Hydroxynonenal, a novel indicator of lipid peroxidation for reperfusion injury of the myocardium," The American Journal of Physiology, vol. 269, pp. H14-H22, 1995.

[9] J. Chen, G. I. Henderson, and G. L. Freeman, "Role of 4hydroxynonenal in modification of cytochrome $c$ oxidase in ischemia/reperfused rat heart," Journal of Molecular and Cellular Cardiology, vol. 33, no. 11, pp. 1919-1927, 2001.

[10] A. L. Furfaro, S. Menini, S. Patriarca et al., "HNE-dependent molecular damage in diabetic nephropathy and its possible prevention by N-acetyl-cysteine and oxerutin," BioFactors, vol. 24, no. 1-4, pp. 291-298, 2005.

[11] T. Grune and K. J. A. Davies, "The proteasomal system and HNE-modified proteins," Molecular Aspects of Medicine, vol. 24, no. 4-5, pp. 195-204, 2003.

[12] B. G. Hill, B. P. Dranka, L. Zou, J. C. Chatham, and V. M. Darley-Usmar, "Importance of the bioenergetic reserve capacity in response to cardiomyocyte stress induced by 4hydroxynonenal," The Biochemical Journal, vol. 424, no. 1, pp. 99-107, 2009.

[13] J. Y. Lee, G. Y. Jung, H. J. Heo et al., “4-Hydroxynonenal induces vascular smooth muscle cell apoptosis through mitochondrial generation of reactive oxygen species," Toxicology Letters, vol. 166, no. 3, pp. 212-221, 2006.

[14] V. B. Patel, C. H. Spencer, T. A. Young, M. O. Lively, and C. C. Cunningham, "Effects of 4-hydroxynonenal on mitochondrial 3-hydroxy-3-methylglutaryl (HMG-CoA) synthase," Free Radical Biology and Medicine, vol. 43, no. 11, pp. 1499-1507, 2007.

[15] V. R. Mali, M. Deshpande, G. Pan, R. A. Thandavarayan, and S. S. Palaniyandi, "Impaired ALDH2 activity decreases the mitochondrial respiration in H9C2 cardiomyocytes," Cellular Signalling, vol. 28, no. 2, pp. 1-6, 2016.

[16] S. J. Watkins, G. M. Borthwick, and H. M. Arthur, "The H9C2 cell line and primary neonatal cardiomyocyte cells show similar hypertrophic responses in vitro," In Vitro Cellular \& Developmental Biology -Animal, vol. 47, no. 2, pp. 125-131, 2011.

[17] B. J. Stuck, M. Lenski, M. Böhm, and U. Laufs, "Metabolic switch and hypertrophy of cardiomyocytes following treatment with angiotensin II are prevented by AMP-activated protein kinase," The Journal of Biological Chemistry, vol. 283, no. 47, pp. 3256232569, 2008.

[18] X. Tan, D.-B. Wang, X. Lu et al., "Doxorubicin induces apoptosis in H9c2 cardiomyocytes: role of overexpressed Eukaryotic translation initiation factor 5A," Biological and Pharmaceutical Bulletin, vol. 33, no. 10, pp. 1666-1672, 2010. 
[19] V. R. Mali, R. Ning, J. Chen, X.-P. Yang, J. Xu, and S. S. Palaniyandi, "Impairment of aldehyde dehydrogenase- 2 by 4-hydroxy-2-nonenal adduct formation and cardiomyocyte hypertrophy in mice fed a high-fat diet and injected with lowdose streptozotocin," Experimental Biology and Medicine, vol. 239, no. 5, pp. 610-618, 2014.

[20] C.-H. Chen, G. R. Budas, E. N. Churchill, M.-H. Disatnik, T. D. Hurley, and D. Mochly-Rosen, "Activation of aldehyde dehydrogenase-2 reduces ischemic damage to the heart," Science, vol. 321, no. 5895, pp. 1493-1495, 2008.

[21] D. Selvakumar, M. J. Drescher, J. R. Dowdall et al., "CNGA3 is expressed in inner ear hair cells and binds to an intracellular Cterminus domain of EMILIN1," Biochemical Journal, vol. 443, no. 2, pp. 463-476, 2012.

[22] S. S. Palaniyandi, Y. Nagai, K. Watanabe et al., "Chymase inhibition reduces the progression to heart failure after autoimmune myocarditis in rats," Experimental Biology and Medicine, vol. 232, no. 9, pp. 1213-1221, 2007.

[23] M. L. Shen, J. J. Lipsky, and S. Naylor, "Role of disulfiram in the in vitro inhibition of rat liver mitochondrial aldehyde dehydrogenase," Biochemical Pharmacology, vol. 60, no. 7, pp. 947-953, 2000.

[24] S. Kumar, V. Kain, and S. L. Sitasawad, "High glucose-induced $\mathrm{Ca}^{2+}$ overload and oxidative stress contribute to apoptosis of cardiac cells through mitochondrial dependent and independent pathways," Biochimica et Biophysica Acta (BBA)-General Subjects, vol. 1820, no. 7, pp. 907-920, 2012.

[25] C. W. Younce, K. Wang, and P. E. Kolattukudy, "Hyperglycaemia-induced cardiomyocyte death is mediated via MCP-1 production and induction of a novel zinc-finger protein MCPIP," Cardiovascular Research, vol. 87, no. 4, pp. 665-674, 2010.

[26] S. S. Palaniyandil, M.-H. Disatnik, L. Sun et al., "Aldehyde dehydrogenase activator attenuates diabetic cardiomyopathy; a role in improving the quality of resident cardiac stem cells?" The FASEB Journal, vol. 24, no. 1, supplement, p. 572.3, 2010.

[27] J. Wang, H. Wang, P. Hao et al., "Inhibition of aldehyde dehydrogenase 2 by oxidative stress is associated with cardiac dysfunction in diabetic rats," Molecular Medicine, vol. 17, no. 34, pp. 172-179, 2011.

[28] M.-C. Desco, M. Asensi, R. Márquez et al., "Xanthine oxidase is involved in free radical production in type 1 diabetes: protection by allopurinol," Diabetes, vol. 51, no. 4, pp. 1118-1124, 2002.

[29] A. San Martín, P. Du, A. Dikalova et al., "Reactive oxygen species-selective regulation of aortic inflammatory gene expression in Type 2 diabetes," American Journal of Physiology-Heart and Circulatory Physiology, vol. 292, no. 5, pp. H2073-H2082, 2007.

[30] E. Shen, Y. Li, Y. Li et al., "Racl is required for cardiomyocyte apoptosis during hyperglycemia," Diabetes, vol. 58, no. 10, pp. 2386-2395, 2009.

[31] E. Gottlieb, S. M. Armour, M. H. Harris, and C. B. Thompson, "Mitochondrial membrane potential regulates matrix configuration and cytochrome c release during apoptosis," Cell Death and Differentiation, vol. 10, no. 6, pp. 709-717, 2003.

[32] Y. Zhang, S. A. Babcock, N. Hu, J. R. Maris, H. Wang, and J. Ren, "Mitochondrial aldehyde dehydrogenase (ALDH2) protects against streptozotocin-induced diabetic cardiomyopathy: role of GSK $3 \beta$ and mitochondrial function," BMC Medicine, vol. 10, article 40, 2012. 


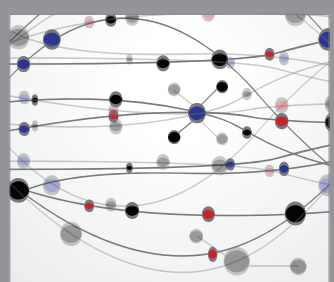

The Scientific World Journal
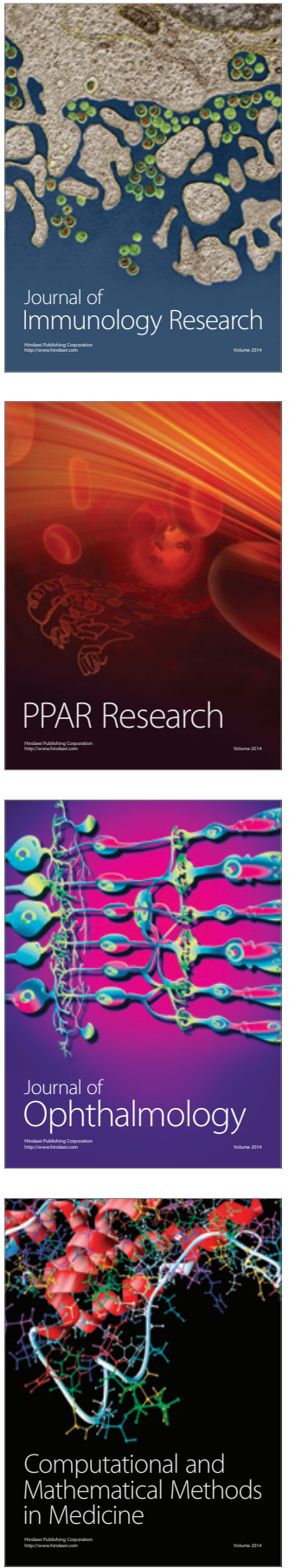

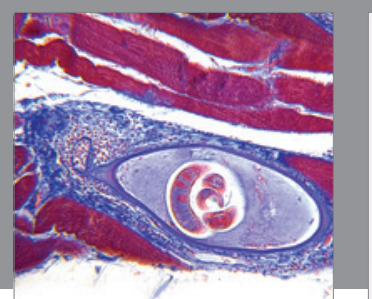

Gastroenterology Research and Practice

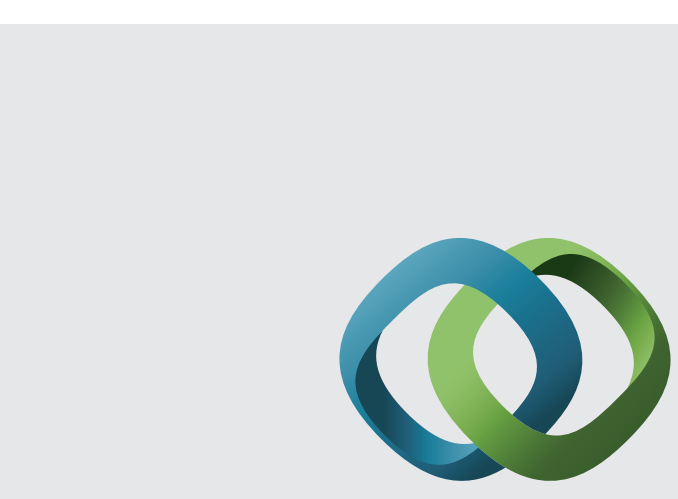

\section{Hindawi}

Submit your manuscripts at

http://www.hindawi.com
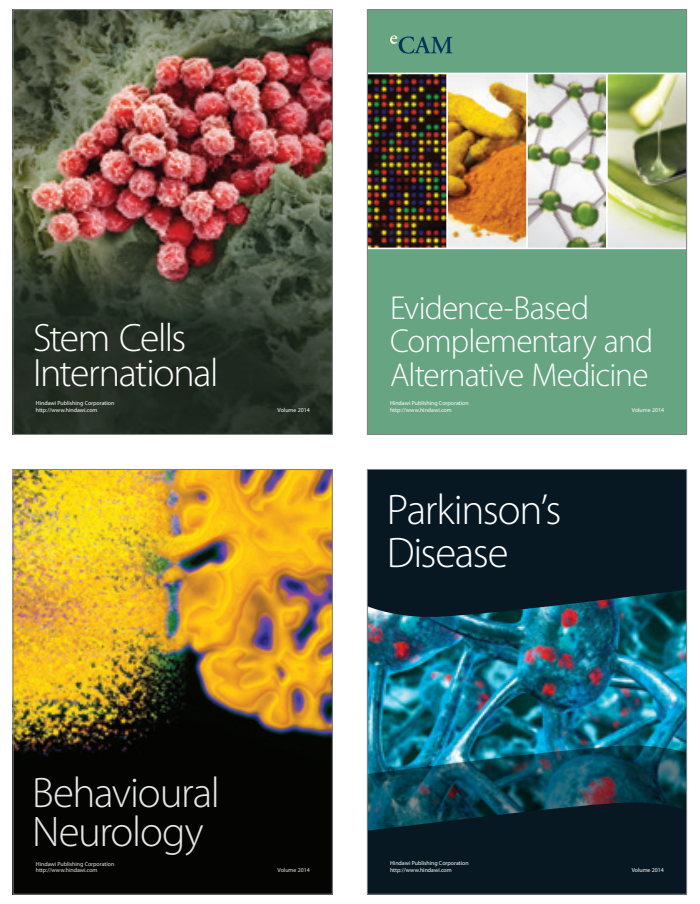
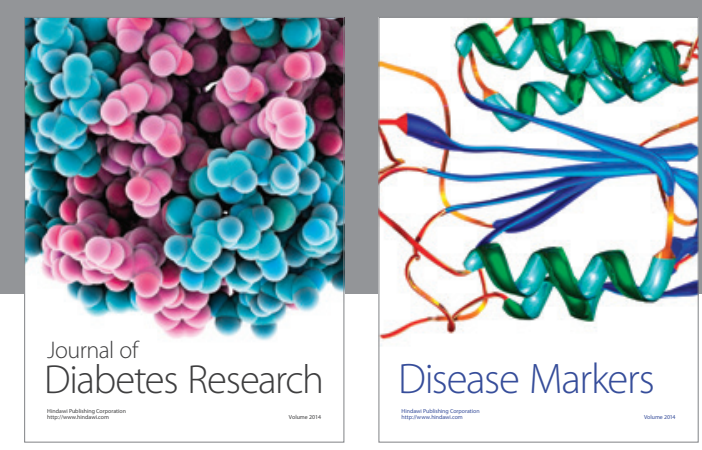

Disease Markers
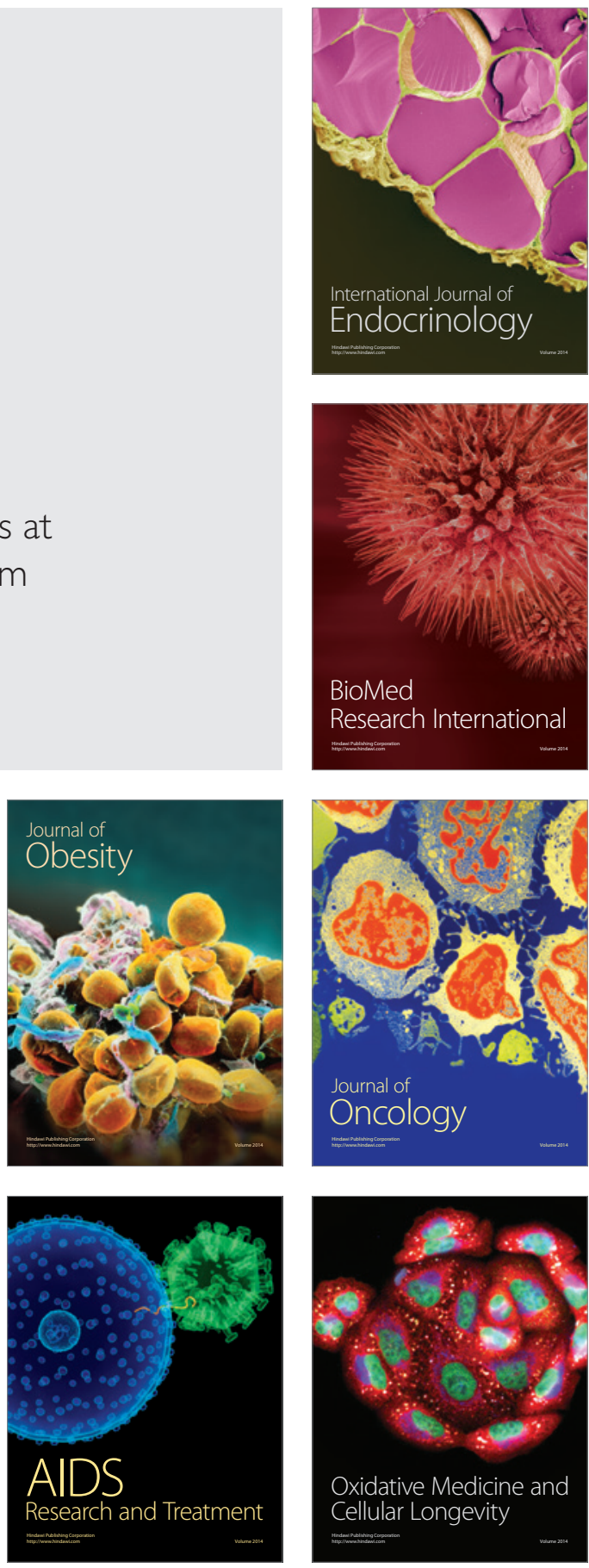